\title{
55 Endurance training and the brain
}

Even during moderate physical activity, cerebral blood circulation is $30 \%$ higher. Rigorous physical activity is the best way to maintain the body's nearly 100 billion brain cells and improve their function by enabling the brain to develop a variety of new synapses. In an adult, every single nerve cell has on average around 1000 such connections to other nerve cells. The total number of these synapses is around $\mathbf{1 0 0}$ trillion. As a result of sport, thought processes become easier, and intelligence, learning and memory are optimally enhanced. The hippocampus, the central region of the brain controlling memory and spatial orientation, undergoes less age-induced atrophy when the elderly regularly engage in adequate physical activity (Smith et al. 2014).

The improved cerebral metabolism leads to greater production of hundreds of chemicals, including nerve growth factors such as brain-derived neurotropic factor (BDNF), which is deficient in depression. Dopamine, serotonin and noradrenaline are some of the many neurotransmitters produced. Of special interest is the up to four-fold increase in the release of endogenous opioids, called endorphins. These are primarily formed in the frontal lobe of the cerebral cortex and the limbic system. Both areas of the brain play a key role in the processing of feelings and in pain suppression. Physical activity confers considerable psychological benefits to well-being, higher self-esteem, the release of pent-up aggression, a distancing from exaggerated problems, mitigating negative feelings and a general resistance to stress. Caution is advised in contact sports because repetitive blows to the head can cause minor damage to the brain (Koerte et al. 2012, McAllister et al. 2014).

Regular exercise stimulates thought processes.

Obesity, often associated with physical inactivity in middle age, increases the risk of dementia ( $\downarrow$ Chapter 38). Insulin resistance, similar to that in diabetics, plays an important role in this. People with diabetes thus have a $60-90 \%$ higher risk of dementia compared to the normal population. According to the findings of current studies, people who are physically active in their youth and engage in lifelong exercise, and those who follow a diet rich in fish, poultry, nuts, salad, fruit and vegetables with limited intake of fat and cow meat ( $\triangleright$ Chapter 32), cannot only reduce minor age-associated cognitive impairment, but also significantly reduce their risk for dementia (Gu et al. 2010, Middleton et al. 2010, Ahlskog et al. 2011, DeFina et al. 2013).

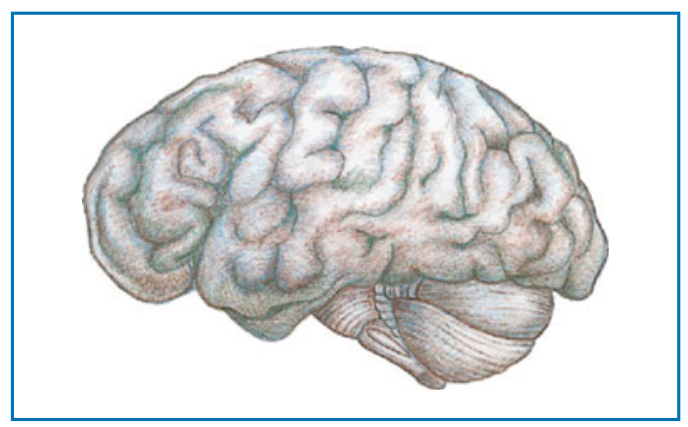

Fig. 55.1 